\title{
Postural Changes During Quiet Stance and Gait Initiation in Slightly Obese Adults
}

\author{
Z. HIRJAKOVÁ ${ }^{1}$, K. ŠUTTOVÁ ${ }^{1}$, J. KIMIJANOVÁ ${ }^{1}$, D. BZDÚŠKOVÁ ${ }^{1}$, F. HLAVAČKA ${ }^{1}$ \\ ${ }^{1}$ Centre of Experimental Medicine Slovak Academy of Sciences, Institute of Normal and \\ Pathological Physiology, Bratislava, Slovakia
}

Received January 24, 2018

Accepted April 25, 2018

On-line September 11, 2018

\section{Summary}

The study is aimed to examine balance control of slightly obese young adults during quiet stance and during gait initiation with and without crossing an obstacle. Forty-four young subjects were divided in two groups: control (BMI $\left.<25 \mathrm{~kg} / \mathrm{m}^{2}\right)$ and slightly obese (BMI from 25 to $35 \mathrm{~kg} / \mathrm{m}^{2}$ ). Center of foot pressure (CoP) and kinematics of fifth lumbar vertebra (L5) were evaluated using a force plate and a motion capture system. During quiet stance with eyes open slightly obese group showed increased mean amplitude and velocity of COP in anterior-posterior direction compared to normal weight subjects. During unloading phase of gait initiation significantly greater and faster lateral CoP shift was observed in slightly obese group compared to normal weight peers. Presence of an obstacle increased amplitude and velocity of the lateral CoP shift similarly in both groups. No BMI-related differences were found on L5 segment during gait initiation, which may indicate that postural control was already successfully performed in feet (CoP). We have shown that increased CoP parameters values and thus increased postural instability during quiet stance and during unloading phase of gait initiation is present not only in morbidly obese, but already in slightly obese subjects.

\section{Key words}

Balance $\bullet$ Body mass index $\bullet$ Center of pressure $\bullet$ Gait $\bullet$ Obstacle

\section{Corresponding author}

Z. Hirjaková, Centre of Experimental Medicine Slovak Academy of Sciences, Institute of Normal and Pathological Physiology, Sienkiewiczova 1, 81371 Bratislava, Slovakia. E-mail: zuzana.hirjakova@savba.sk

\section{Introduction}

Increased body mass index (BMI) has been linked with an increased risk of a number of significant health issues including musculoskeletal problems (Messier et al. 2005). It has been shown that obesity can lead to poorer balance control (Chiari et al. 2002, McGraw et al. 2000). Teasdale et al. (2007) showed that postural stability, i.e. center of foot pressure $(\mathrm{CoP})$ speed and range in anterior-posterior (AP) and medio-lateral (ML) direction, improved in obese and morbid obese men after losing weight. A strong linear relationship between the weight loss and the improvement in balance control supports the idea that weight could be an important predictor of postural stability (Hue et al. 2007). Besides increased postural instability during stance, obese individuals have been shown to walk with a reduced step length, cadence and walking speed and with increased step width, longer stance phase and double support period during a gait cycle (Spyropoulos et al. 1991). Generally, instant instability increases during gait because body weight is put on one side of the leg and the body moves to maintain center of gravity ( $\mathrm{CoG}$ ) within base of support to reduce such instability. The gait initiation is the transient phase between quiet posture and steady-state walking (Elble et al. 1994). Most locomotion-related falls occur while initiating gait (Cau et al. 2014, Uemura et al. 2012), therefore it is often used for identifying postural control deficiencies (Ruget et al. 2008, Martin et al. 2002, Halliday et al. 1998). Gait initiation is typically associated with anticipatory postural adjustments (APAs) and occurs prior to gross segmental movement and 
stability boundary changes of the first step (Cau et al. 2014). APAs reconfigure the position of the center of mass $(\mathrm{CoM})$ with respect to the CoP (Brenière and Do 1986) to stabilize posture and assist motor performance (Gélat et al. 2011, Ruget et al. 2008, MacKinnon et al. 2007). According to Ruget et al. (2008) APAs are characterized in lateral direction by a force exerted by the moving leg onto the ground (thrust), followed by an unloading of the stepping leg and completed by an adjustment corresponding to a slow $\mathrm{CoP}$ shift toward a supporting leg. Other studies (Honeine et al. 2016, Winter 1995, Carlsoo 1966) showed that the initial displacement of the CoP in the direction of the swing leg is caused by unloading the stance leg and loading the swing leg. This is caused by a hip abduction from the swing side accompanied by a small knee flexion from the stance side (Honeine et al. 2016). Previous evaluation of the anticipatory postural adjustments in slightly obese subjects was mostly focused on morbidly obese subjects with mean BMI greater than $35 \mathrm{~kg} / \mathrm{m}^{2}$ (Cau et al. 2014, Menegoni et al. 2009, Colné et al. 2008). Little is known about postural stability and APAs parameters (amplitude and velocity) in slightly obese subjects with the BMI lower than $35 \mathrm{~kg} / \mathrm{m}^{2}$. Especially, APAs parameters prior to stepping over an obstacle were not evaluated in these subjects. Even a low height obstacle such as sidewalk bricks may increase the postural instability during gait initiation. Greater and faster motion of body segments while negotiating an obstacle will result in greater and faster movement of the whole body (Chou and Draganich 1997, Patla and Rietdyk 1993). Yiou et al. (2016) showed that the anticipatory peak of CoP ML shift, the initial CoM ML velocity and the duration of swing phase of gait increased with obstacle height, but not with obstacle distance. According to these authors the central nervous system (CNS) in healthy people is able to precisely predict the potential instability elicited by obstacle clearance and maintain optimal postural stability. CNS is able to scale and coordinate the AP and ML components of APAs according to obstacle constrains. Inappropriate body segment coordination in response to the obstacle crossing will likely perturb balance maintenance in the frontal plane and may cause fall to the side (Greenspan et al. 1994, Nevitt and Cummings 1993). According to Chou et al. (2003) stepping over an obstacle results in significantly greater ranges of motion of CoM in the AP direction. The range of motion and peak velocity of the $\mathrm{CoM}$ in the ML direction is less likely to be affected when negotiating obstacles in healthy individuals. Therefore, control of ML stability during gait initiation may be used as functional indicator to identify persons who are at greater risk of imbalance. Identifying early signs of altered postural reactions in slightly obese subject may be beneficial for exercise training and prevention programs to improve postural stability and minimize the risk of falling.

The aim of this study was to investigate postural stability during quiet stance with eyes open and during the gait initiation in normal weight subjects and slightly obese subjects with the BMI lower than $35 \mathrm{~kg} / \mathrm{m}^{2}$. We hypothesize that 1 ) postural stability during quiet stance will be compromised in slightly obese subjects compared to normal weight subjects; 2) postural parameters in lateral direction during unloading phase of gait initiation will be modulated in slightly obese subjects, especially prior to stepping over an obstacle.

\section{Methods}

Forty-four subjects participated in the study. According to their body mass index values, they were divided into two groups: control (normal weight subject with the BMI lower than $25 \mathrm{~kg} / \mathrm{m}^{2}$ ) and slightly obese group (subjects with the BMI from 25 to $35 \mathrm{~kg} / \mathrm{m}^{2}$ ) (Table 1). Participants were free of any neurological or musculoskeletal disorders. The study was approved by the local ethics committee and the written consent in agreement with the Declaration of Helsinki was obtained from all participants.

Table 1. Characteristics of control and slightly obese group.

\begin{tabular}{|c|c|c|c|c|}
\hline & n (male/female) & Age (years) & BMI $\left(\mathrm{kg} / \mathrm{m}^{2}\right)$ & Stance width $(\mathrm{cm})$ \\
\hline Control & $22(13 / 9)$ & $32.2 \pm 1.3$ & $22.2 \pm 1.2$ & $17.0 \pm 0.7$ \\
\hline Slightly obese & $22(13 / 9)$ & $32.5 \pm 1.3$ & $32.0 \pm 0.9^{* * *}$ & $19.6 \pm 0.7^{*}$ \\
\hline
\end{tabular}

Data are presented as mean $\pm \mathrm{SEM} ; * \mathrm{p}<0.05, * * * \mathrm{p}<0.001$, control vs. slightly obese. 
The protocol consisted of three conditions. In the first static condition subjects stood quietly on firm surface with eyes open (EO) for $50 \mathrm{~s}$. They stood with a self-selected stance width which remained consistent also for all dynamic trials with gait initiation (GI). Since subjects in the slightly obese group stood with significantly greater stance width (Table 1), all CoP parameters of this group were normalized to the averaged stance width of the control group.

In the GI condition, the participants were instructed to initiate gait with their right leg after hearing a sound signal and make few steps. In the GIO condition, they were asked to cross an obstacle (height $\mathrm{x}$ width $\mathrm{x}$ length; $13 \times 33 \times 7 \mathrm{~cm}$ ) situated in front of them. The distance of the obstacle remained unchanged for all participants. Each gait initiation trial was repeated five times. In EO condition and in the beginning of each gait initiation trial subjects stood on a custom-made force platform with 3 inbuilt force transducers embedded in the laboratory floor (for more details see Hirjaková et al. 2017). Displacements of CoP in AP and ML directions were sampled at the frequency of $100 \mathrm{~Hz}$ and processed with a $2^{\text {nd }}$-order low-pass Butterworth filter with a cut-off frequency of $5 \mathrm{~Hz}$. A motion capture system $\left(\mathrm{BTS}^{\circledR}\right.$ Smart DX, Italy) with six cameras with sampling frequency of $100 \mathrm{~Hz}$ was used for recording of body segment kinematics. One reflective marker was placed on the level of fifth lumbar vertebra (L5), the approximate level of CoM.

The obtained data were analyzed with MATLAB software (MATLAB, Mathworks, Inc., Natick, MA, USA). Four parameters of CoP were evaluated in EO condition: amplitude in AP (Aap) and ML (Aml) direction and velocity in AP (Vap) and $\mathrm{ML}$ (Vml) direction. Amplitudes of $\mathrm{CoP}$ displacement were calculated as multiplications of $\mathrm{SD}(\mathrm{CoP})$ : Aap = $3.92 * \mathrm{SD}$ (CoPap) and Aml $=3.92 * \mathrm{SD}$ (CoPml). Assuming $\mathrm{CoP}$ as normally distributed, Aap and Aml represent $95 \%$ of total CoP amplitudes (Hlavačka et al. 1990). Mean velocity in each direction was calculated according to Prieto et al. (1996) as the average velocity of the CoP in the particular direction. CoP data from five repetitions of GI and GIO conditions were averaged, afterwards two parameters in ML direction were evaluated: 1) peak to peak amplitude (App) - the amplitude in ML direction between maximal position of CoP in the swing side (right) and the maximal position of $\mathrm{CoP}$ in the stance side (left). App represents the lateral shift toward the stance foot during the unloading phase of
APAs; 2) peak to peak velocity (Vpp) - the velocity in ML direction between maximal position of $\mathrm{CoP}$ in the swing side (right) and the maximal position of $\mathrm{CoP}$ in the stance side (left). Vpp represents the maximal velocity of the lateral shift toward the stance foot during the unloading phase of gait initiation. Kinematic data from the L5 marker were processed in the same way as the CoP data. Peak to peak amplitude (App) and peak to peak velocity (Vpp) of the L5 marker were evaluated likewise.

Statistical analysis was performed with SPSS software (SPSS Inc., Chicago, IL, USA). The normality was examined using Shapiro-Wilk test. Since the analyzed parameters were normally distributed repeated measures ANOVA were performed. In the static condition (EO) ANOVA was used to determine the effect of group related to different BMI, the effect of sway direction and their interaction on CoP amplitude and CoP velocity. In dynamic conditions (GI, GIO) repeated measures ANOVA were used to determine the effect of group, obstacle and their interaction on CoP and kinematic (L5) parameters. Post hoc pairwise comparisons with Bonferroni adjustments were conducted for detailed exploration in cases of significant effect of the main factors. Student's t-test was used for exploration of differences between groups in GIO condition while considering relative data (GI values as $100 \%$ ). The significance level was set at $\mathrm{p}<0.05$.

\section{Results}

In static EO condition, repeated measures ANOVA revealed significant effect of the main factors: group, sway direction and their interaction on CoP amplitude $\left(\mathrm{F}_{(1,42)}=7.93, \mathrm{p}=0.007 ; \mathrm{F}_{(1,42)}=83.94, \mathrm{p}<0.001\right.$; $\mathrm{F}_{(1,42)}=12.04, \mathrm{p}=0.001$, respectively) and on CoP velocity $\left(\mathrm{F}_{(1,42)}=10.82, \quad \mathrm{p}=0.002 ; \quad \mathrm{F}_{(1,42)}=116.93, \quad \mathrm{p}<0.001\right.$; $\mathrm{F}_{(1,42)}=15.78, \mathrm{p}<0.001$, respectively). Amplitude and velocity in both directions significantly increased in slightly obese group compared to control group (Fig. 1). Pairwise comparisons also showed that amplitude $(\mathrm{p}<0.001)$ and velocity $(\mathrm{p}<0.001)$ in AP direction significantly increased compared to ML direction in both groups.

In dynamic conditions of gait initiation, repeated measures ANOVA proved that the group and the obstacle $\left(\mathrm{F}_{(1,42)}=6.08, \mathrm{p}=0.018 ; \mathrm{F}_{(1,42)}=61.23, \mathrm{p}<0.001\right.$, respectively) had significant effect on CoP peak to peak amplitude (App) (Fig. 2, Fig. 3A). App significantly increased in slightly obese group compared to control in 

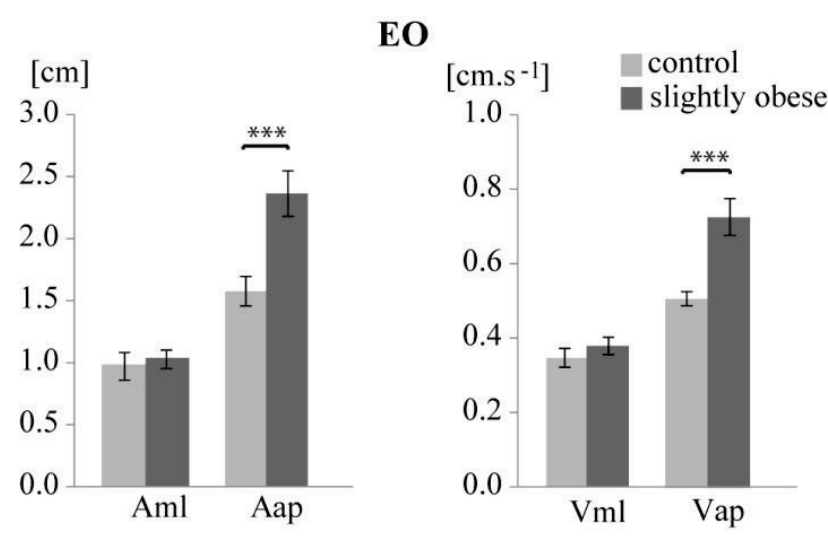

Fig. 1. The mean values of CoP amplitude and velocity in AP and ML direction in control (grey) and slightly obese (black) groups during quiet stance with eyes open (EO). Data are presented as mean \pm SEM; *** $\mathrm{p}<0.001$.

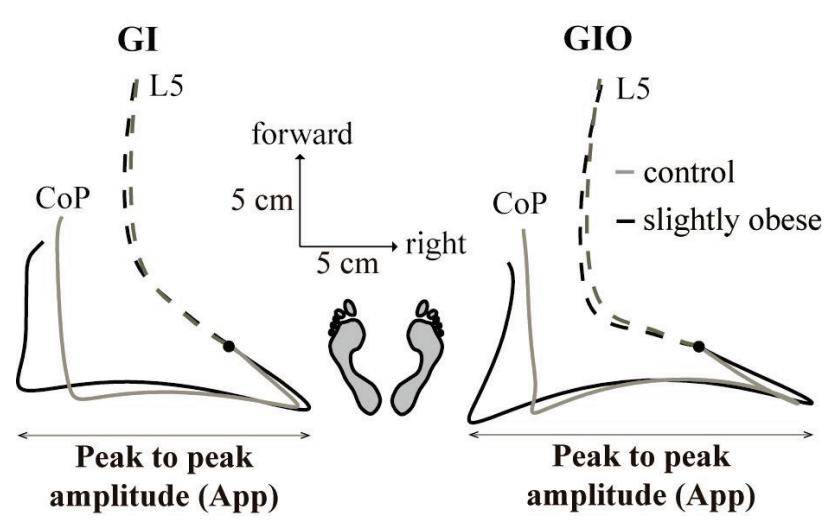

Fig. 2. The group average of CoP (solid lines) and L5 marker (dashed lines) trajectories for control (grey) and slightly obese group (black) in gait initiation (GI) and gait initiation with obstacle (GIO) conditions. Black dots mark the start points.

both GI and GIO conditions (Fig. 3B). Pairwise comparisons with Bonferroni adjustment showed that App significantly increased $(p<0.001)$ while crossing the obstacle compared to gait initiation without the obstacle in both groups. However, considering relative data (GI values as $100 \%$ ), the increase of App in GIO condition compared to GI was very similar in control and slightly obese groups $(113.8 \%$ vs. $115.9 \%, \mathrm{p}=0.610$, respectively).

Statistical analysis of kinematic (L5) data revealed significant effect of the obstacle $\left(\mathrm{F}_{(1,42)}=27.95\right.$, $\mathrm{p}<0.001)$ but no effect of group on peak to peak amplitude (App). Post hoc pairwise comparisons revealed significantly increased App of L5 while crossing the obstacle compared to gait initiation without the obstacle in both groups $(\mathrm{p}<0.001)$.

Repeated measures ANOVA proved significant effect of group and the obstacle $\left(\mathrm{F}_{(1,42)}=7.78, \mathrm{p}=0.008\right.$; $\mathrm{F}_{(1,42)}=67.95, \mathrm{p}<0.001$, respectively) on CoP peak to peak
A

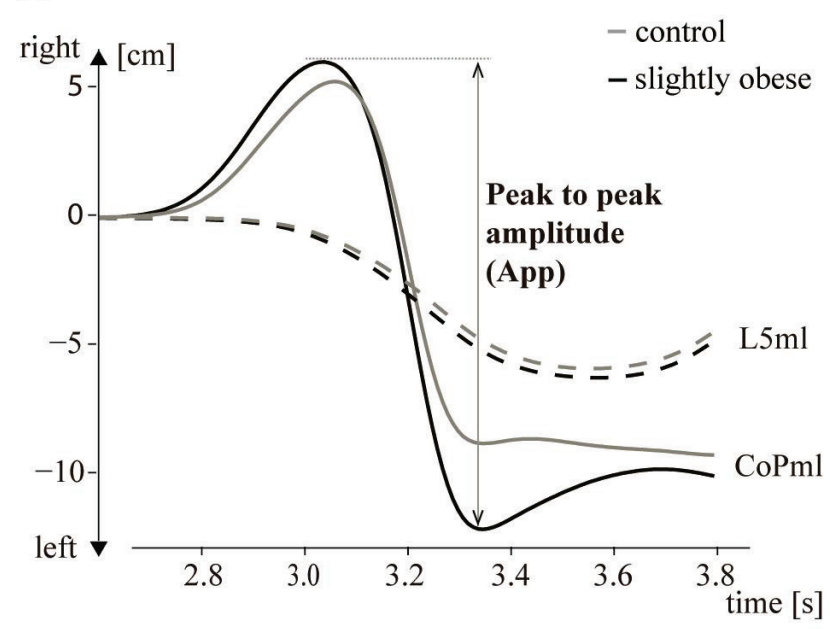

B

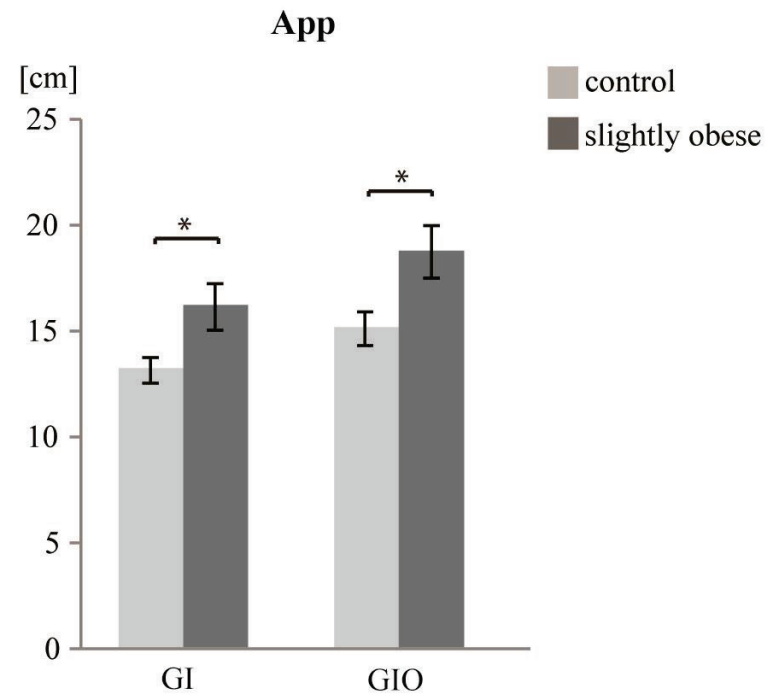

Fig. 3. A. The group average of CoP (solid lines) and L5 marker (dashed lines) traces in lateral direction for control (grey) and slightly obese (black) groups during gait initiation with obstacle (GIO). Double arrow represents the peak to peak amplitude (App) calculated from CoP signal in ML direction. B. The mean values of CoP peak to peak amplitude in ML direction in control (grey) and slightly obese (black) groups during gait initiation without (GI) and with obstacle (GIO). Data are presented as mean \pm SEM; $* \mathrm{p}<0.05$.

velocity (Vpp) (Fig. 4A). Vpp significantly increased in slightly obese subjects compared to control in both conditions. Similarly as for App, Vpp significantly increased $(\mathrm{p}<0.001)$ while crossing the obstacle compared to gait initiation without obstacle in both groups (Fig. 4B). However, relative data (GI values as $100 \%$ ) showed there was no difference in the Vpp increase in control group compared to slightly obese group ( $136.1 \%$ vs. $138.8 \%, \mathrm{p}=0.792$, respectively). Statistical analysis of kinematic (L5) data showed no effect of the obstacle or the group on peak to peak velocity (Vpp) parameter. 

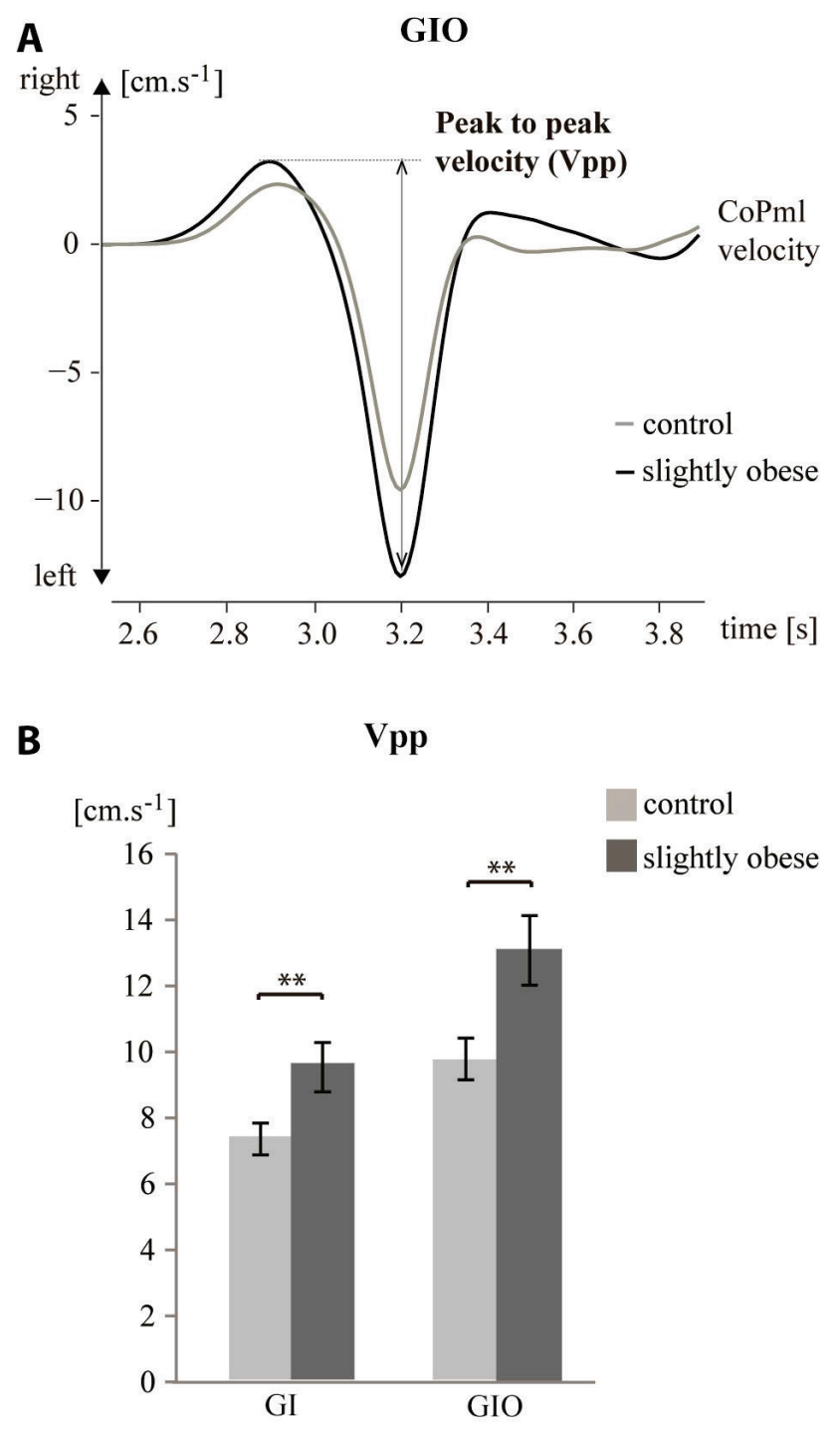

Fig. 4. A. The group average of CoP velocity in lateral direction in control (grey) and slightly obese (black) groups during gait initiation with obstacle (GIO). Double arrow represents the peak to peak velocity (Vpp) calculated from CoP signal in ML direction. B. The mean values of CoP peak to peak velocity in ML direction in control (grey) and slightly obese (black) groups during gait initiation without (GI) and with obstacle (GIO). Data are presented as mean $\pm \mathrm{SEM} ; * \mathrm{p}<0.05$, ** $\mathrm{p}<0.01$.

\section{Discussion}

The aim of this study was to clarify balance control in slightly obese young subjects during quiet stance and during dynamic unloading phase of gait initiation. In the quiet stance with eyes open slightly obese subjects showed increased amplitude and velocity of $\mathrm{CoP}$ in anterior-posterior direction compared to normal weight subjects. In dynamic conditions of gait initiation slightly obese subjects manifested significantly greater lateral $\mathrm{CoP}$ peak to peak amplitude and velocity during unloading phase of gait initiation. No BMI-related differences were observed on lower trunk (L5) parameters.

It is known that obese people usually stand with wider stance, which is associated with large reduction in lateral body motion (Day et al. 1993). Also in our study the slightly obese group showed significantly wider stance, therefore all evaluated parameters were normalized to stance width of the normal weight group. Nevertheless, significant increase of CoP parameters in both static and dynamic conditions was still observed in slightly obese group compared to control group.

During quiet stance with eyes open (EO) slightly obese subjects showed larger $\mathrm{CoP}$ amplitude and faster CoP velocity in anterior-posterior direction compared to normal weight subjects. An increase in body weight has been previously correlated to decrease in postural stability because the balance control system is less sensitive to regulate body sway oscillations (Hue et al. 2007). BMI higher than $35 \mathrm{~kg} / \mathrm{m}^{2}$ has been associated with higher CoP speed and increased RMS in both directions (Menegoni et al. 2009, Hue et al. 2007). The effectiveness of the postural control system is generally related to the magnitude (amplitude) of the CoP displacement, while CoP speed is associated with activity necessary to maintain postural stability (Hue et al. 2007, Prieto et al. 1996). We have proved that CoP velocity and $\mathrm{CoP}$ amplitude increased already in slightly obese subjects with BMI from 25 to $35 \mathrm{~kg} / \mathrm{m}^{2}$. Higher CoP velocity and amplitude may be therefore considered as valuable indicators of balance instability and risk of falling (Maki et al. 1994, Fernie et al. 1982) already in slightly obese subjects not only in morbidly obese (Cau et al. 2014, Menegoni et al. 2009, Colné et al. 2008). During static EO condition significant differences between groups were found only in AP direction. Postural sway is usually increased in AP direction compared to ML direction during quiet stance since the body acts as an inverted pendulum with joint rotation primarily around the ankle. An inverted pendulum operates with the ankle strategy in AP direction while the hip strategy in ML direction is activated as the postural challenge increases (Runge et al. 1999). BMI lower than $35 \mathrm{~kg} / \mathrm{m}^{2}$ probably did not represent demands large enough for activation of the hip strategy. Also wider stance of slightly obese subjects probably contributed in stabilization of lateral body motion (Day et al. 1993). These may be reasons why differences between groups were found only in AP direction.

While there were no BMI-related changes in ML 
direction during static condition of quiet stance (EO), significant lateral differences between groups were found in dynamic conditions of gait initiation. Destabilizing effect of higher BMI was observed during the unloading phase of gait initiation with and also without crossing the obstacle. Slightly obese subjects loaded the weight of their body from one leg to another with greater and faster CoP shift represented by increased peak to peak amplitude and velocity (Fig. 2, Fig. 3 and Fig. 4). This is in line with previous study when the gait initiation in obese subjects $\left(\mathrm{BMI}>37 \mathrm{~kg} / \mathrm{m}^{2}\right)$ was represented by a higher excursion of lateral CoP shift toward the stance foot (Cau et al. 2014). The center of gravity shift toward a new base of support is initiated by the generation of propulsive forces. The strategy used to transfer center of gravity onto supporting leg requires inertial forces to be overcome. Greater body weight supported by the moving leg is associated with greater inertial forces necessary to transit from a bipedal stance to a single limb stance in obese subjects (Rogers and Pai 1990). Lateral CoP shift was faster and greater while crossing the obstacle compared to gait initiation without the obstacle in both groups. Increased App and Vpp parameters in slightly obese group in GIO condition could be explained by the differences of stiffness associated with higher BMI. When crossing an obstacle young healthy adults scale and coordinate the AP and ML components of APAs in order to take into account the biomechanical properties of their body (Yiou et al. 2016).

In contrast to $\mathrm{CoP}$ parameters, the kinematic analysis of L5 marker did not show the destabilizing effect of higher BMI on lateral shift of lower trunk during gait initiation. Possible explanation for the invariant lateral L5 (CoM) motion may be the fact that balance control is generally performed in feet (Chou et al. 2003). This may indicate that control strategy of slightly obese adults successfully maintained the line of progression within the base of support to avoid imbalance of lower trunk segment (L5). It is also possible that the lack of statistical effect on the L5 marker may be due to the difference in initial stance width. During GI condition, the ML displacement of the CoP is caused mainly by hip abduction and knee flexion (Honeine et al. 2016). Feet muscles also contribute to ML displacement of $\mathrm{CoP}$ and CoP displacement allows gravity to displace the center of gravity (Carlsoo 1966). Therefore it is possible that larger stance width could cause the greater knee flexion in slightly obese subjects. To minimize the effect of wider stance in slightly obese subjects in our study, the normalization process was used.

In summary, BMI-related differences were found during static EO condition with slightly obese subjects manifesting increased $\mathrm{CoP}$ amplitude and velocity in AP direction. In dynamic condition slightly obese subjects showed greater and faster $\mathrm{CoP}$ shift during unloading phase of gait initiation compared to normal weight adults. The CoP shift was faster and greater while crossing the obstacle in both groups. There were no BMIrelated differences in lateral shift of lower trunk (L5) during unloading phase of gait initiation. We proved that increased postural instability during stance and also during gait initiation is present not only in morbidly obese adults, but also in slightly obese with BMI lower than $35 \mathrm{~kg} / \mathrm{m}^{2}$.

\section{Conflict of Interest}

There is no conflict of interest.

\section{Acknowledgements}

This work was supported by Slovak grant agency VEGA No. 2/0094/16, 1/0824/17 and by the project Cekomat II ITMS 26240120020 .

\section{References}

BRENIÈRE Y, DO MC: When and how does steady state gait movement induced from upright posture begin? J Biomech 19: 1035-1040, 1986.

CARLSOO S: The initiation of walking. Acta Anat (Basel) 65: 1-9, 1966.

CAU N, CIMOLIN V, GALLI M, PRECILIOS H, TACCHINI E, SANTOVITO C, CAPODAGLiO P: Center of pressure displacements during gait initiation in individuals with obesity. J Neuroeng Rehabil 11: 82, 2014.

CHIARI L, ROCCHI L, CAPPELLO A: Stabilometric parameters are affected by anthropometry and foot placement. Clin Biomech 17: 666-677, 2002.

CHOU LS, DRAGANICH LF: Stepping over an obstacle increases the motions and moments of the joints of the trailing limb in young adults. $J$ Biomech 30: 331-337, 1997. 
CHOU LS, KAUFMAN KR, HAHN ME, BREY RH: Medio-lateral motion of the center of mass during obstacle crossing distinguishes elderly individuals with imbalance. Gait Posture 18: 125-133, 2003.

COLNÉ P, FRELUT ML, PÉRÈS G, THOUMIE P: Postural control in obese adolescents assessed by limits of stability and gait initiation. Gait Posture 28: 164-169, 2008.

DAY BL, STEIGER MJ, THOMPSON PD, MARSDEN CD: Effect of vision and stance width on human body motion when standing: implications for afferent control of lateral sway. $J$ Physiol 469: 479-499, 1993.

ELBLE RJ, MOODY C, LEFFLER K, SINHA R: The initiation of normal walking. Mov Disord 9: 139-146, 1994.

FERNIE GR, GRYFE CI, HOLLIDAY PJ, LLEWELLYN A: The relationship of postural sway in standing to the incidence of falls in geriatric subjects. Age Ageing 11: 11-16, 1982.

GÉLAT T, COUDRAT L, LE PELLEC A: Gait initiation is affected during emotional conflict. Neurosci Lett 497 : 64-67, 2011.

GREENSPAN SL, MYERS ER, MAITLAND LA, RESNICK NM, HAYES WC: Fall severity and bone mineral density as risk factors for hip fracture in ambulatory elderly. J Am Med Assoc 271: 128-133, 1994.

HALLIDAY SE, WINTER DA, FRANK JS, PATLA AE, PRINCE F: The initiation of gait in young, elderly and Parkinson's disease subjects. Gait Posture 8: 8-14, 1998.

HIRJAKOVÁ Z, NEUMANNOVÁ K, KIMIJANOVÁ J, ŠUTTOVÁ K, JANURA M, HLAVAČKA F: Breathing changes accompanying balance improvement during biofeedback. Neurosci Lett 651: 30-35, 2017.

HLAVAČKA F, KUNDRÁT J, KRIŽKOVÁ M, BAČOVÁ E: Physiologic range of stabilometry values obtained in the upright posture using a computer (in Slovak). Cesk Neurol Neurochir 53: 107-113, 1990.

HONEINE JL, SCHIEPPATI M, CRISAFULLI O, DO MC: The neuro-mechanical processes that underlie goal-directed medio-lateral APA during gait initiation. Front Hum Neurosci 10: 445, 2016.

HUE O, SIMONEAU M, MARCOTTE J, BERRIGAN F, DORÉ J, MARCEAU P, MARCEAU S, TREMBLAY A, TEASDALE N: Body weight is a strong predictor of postural stability. Gait Posture 26: 32-38, 2007.

MACKINNON CD, BISSIG D, CHIUSANO J, MILLER E, RUDNICK L, JAGER C, ZHANG Y, MILLE ML, ROGERS MW: Preparation of anticipatory postural adjustments prior to stepping. J Neurophysiol 97: 43684379, 2007.

MAKI BE, HOLLIDAY PJ, TOPPER AK: A prospective study of postural balance and risk of falling in an ambulatory and independent elderly population. J Gerontol 49: M72-M84, 1994.

MARTIN M, SHINBERG M, KUCHIBHATLA M, RAY L, CAROLLO JJ, SCHENKMAN ML: Gait initiation in community-dwelling adults with Parkinson disease: comparison with older and younger adults without disease. Phys Ther 82: 566-577, 2002.

MCGRAW B, MCCLENAGHAN BA, WILLIAMS HG, DICKERSON J, WARD DS: Gait and postural stability in obese and nonobese prepubertal boys. Arch Phys Med Rehabil 81: 484-489, 2000.

MENEGONI F, GALLI M, TACCHINI E, VISMARA L, CAVIGIOLI M, CAPODAGLIO P: Gender-specific effect of obesity on balance. Obesity (Silver Spring) 17: 1951-1956, 2009.

MESSIER SP, GUTEKUNST DJ, DAVIS C, DEVITA P: Weight loss reduces knee-joint loads in overweight and obese older adults with knee osteoarthritis. Arthritis Rheumatol 52: 2026-2032, 2005.

NEVITT MC, CUMMINGS SR: Type of fall and risk of hip and wrist fractures: the study of osteoporotic fractures. The Study of Osteoporotic Fractures Research Group. J Am Geriatr Soc 41: 1226-1234, 1993.

PATLA AE, RIETDYK S: Visual control of limb trajectory over obstacles during locomotion: effect of obstacle height and width. Gait Posture 1: 45-60, 1993.

PRIETO TE, MYKLEBUST JB, HOFFMANN RG, LOVETT EG, MYKLEBUST BM: Measures of postural steadiness: differences between healthy young and elderly adults. IEEE Trans Biomed Eng 43: 956-966, 1996.

ROGERS MW, PAI YC: Dynamic transitions in stance support accompanying leg flexion movements in man. Exp Brain Res 81: 398-402, 1990.

RUGET H, BLOUIN J, TEASDALE N, MOUCHNINO L: Can prepared anticipatory postural adjustments be updated by proprioception? Neuroscience 155: 640-648, 2008.

RUNGE CF, SHUPERT CL, HORAK FB, ZAJAC FE: Ankle and hip postural strategies defined by joint torques. Gait Posture 10: 161-170, 1999. 
SPYROPOULOS P, PISCIOTTA JC, PAVLOU KN, CAIRNS MA, SIMON SR: Biomechanical gait analysis in obese men. Arch Phys Med Rehabil 72: 1065-1070, 1991.

TEASDALE N, HUE O, MARCOTTE J, BERRIGAN F, SIMONEAU M, DORÉ J, MARCEAU P, MARCEAU S, TREMBLAY A: Reducing weight increases postural stability in obese and morbid obese men. Int J Obes $\mathbf{3 1}$ : 153-160, 2007.

UEMURA K, YAMADA M, NAGAI K, TANAKA B, MORI S, ICHIHASHI N: Fear of falling is associated with prolonged anticipatory postural adjustment during gait initiation under dual-task conditions in older adults. Gait Posture 35: 282-286, 2012.

WINTER D: Human balance and posture control during standing and walking. Gait Posture 3: 193-214, 1995.

YIOU E, ARTICO R, TEYSSEDRE CA, LABAUNE O, FOURCADE P: Anticipatory postural control of stability during gait initiation over obstacles of different height and distance made under reaction-time and self-initiated instructions. Front Hum Neurosci 10: 449, 2016. 